\title{
Two Cases of Pure Hair Nail Ectodermal Dysplasia in Two Yemeni Siblings
}

Keywords: Skin; Hair; Nail; Ectodermal dysplasia

Abstract

Ectodermal dysplasias (ED) are a rare group of genodermatoses affecting tissues of ectodermal origin namely: hair, nails, teeth, and sweat glands. Pure hair, nail ectodermal dysplasias (PHNED) are very rare, and involve only hair and nails, while other ectodermal structures are intact. To date only 20 cases have been reported in the world literature. We report two cases of PHNED in a 15 years old Yemeni boy, and his 2 months old sister, who were born with total absence of hairs and dystrophic 20 nails. All other family members were not affected.

\section{Introduction}

Pure hair-nail ectodermal dysplasias (PHNED) are very rare subtype of Ectodermal dysplasias (ED), involving only hair and nails, while other ectodermal structures (teeth and sweat glands) are intact. The first case of ED was reported by Thurnam in 1848, while Weech coined the term ED in 1929 [1,2]. To date only 20 cases of PHNED have been reported worldwide.

\section{Case 1}

A 15 years old Yemeni boy presented with total alopecia involving the scalp, brows, and lashes and distal 20 nail dystrophy since birth (Figure 1a). On close inspection of the scalp, small black hairs can be seen within the hair follicles (Figure 1b). All 20 nails were distally dystrophic and sloped (Figures 1c and 1d). Teeth and sweat glands were not affected. The histological examination of the scalp showed severely reduced number of structurally disorganized hair follicles (Figure 1e). The patient was otherwise healthy. His parents and his 11, 9 years old brothers, and 5 years old sister, were not affected, suggesting an autosomal recessive mode of inheritance (Figure 2).

\section{Case 2}

The two months old sister of case 1, presented with similar albeit milder manifestations as her brother (Figures 3a-3d).

\section{Discussion}

EDs are rare group of genodermatoses affecting tissues of ectodermal origin namely: hair, nails, teeth, and sweat glands. Freire-Maia and Pinheiro proposed the first classification system for ectodermal dysplasias in 1982, with additional updates in 1994 and $2001[3,4]$. PHNED are very rare subtype of ED. They can be inherited as autosomal recessive and autosomal dominant pattern, with highly variable clinical expression [5,6]. A summary of clinical and genetic finding in previously reported cases is shown (Table 1). Affected individuals are usually born devoid of hairs of the scalp, brows, and lashes. All 20 nails are distally dystrophic and sloping, and they never need to be trimmed. To date only 20 cases of PHNED

\section{Journal of}

\section{Clinical \& Investigative} Dermatology

\section{Mohammad Ali Alshami ${ }^{1^{*}}$, Mona Jameel Mohana ${ }^{1}$ and Ahlam Mohammad Alshami}

${ }^{1}$ Dermatology Department, Faculty of medicine, and health sciences, Sana'a University, Sana'a, Yemen

${ }^{2}$ Faculty of Dentistry, Sana'a University, Sana'a, Yemen

*Address for Correspondence

Mohammad Ali Alshami, Dermatology Department, Faculty of medicine, and health sciences, Sana'a University, Sana'a, Yemen, Tel: 00967733760082; E-mail: mohammadalshami62@gmail.com

Submission: 08 June, 2016

Accepted: 02 July, 2016

Published: 06 July, 2016

Copyright: () 2016 Alshami MA, et al. This is an open access article distributed under the Creative Commons Attribution License, which permits unrestricted use, distribution, and reproduction in any medium, provided the original work is properly cited.

Reviewed \& Approved by: Dr. Hyun Sun Park, Department of Dermatology, Seoul National University, Korea

have been reported worldwide. Mutation in the KRT85 gene was found in some patients, while mutations either in the COXC13 or KRT74 genes were found in others [7-9]. Keratin 85 and keratin 74 belong to keratin type II, while COXC13 is a transcription regulator of keratin and keratin-associated protein genes. Keratins are important intermediate filaments of keratinocytes of the hair and nails that give them the ability to resist mechanical trauma. They are classified into
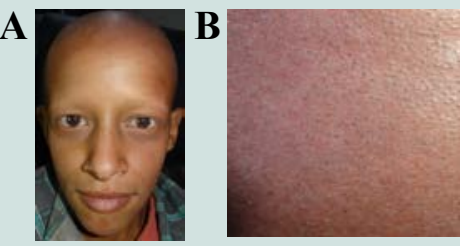

C

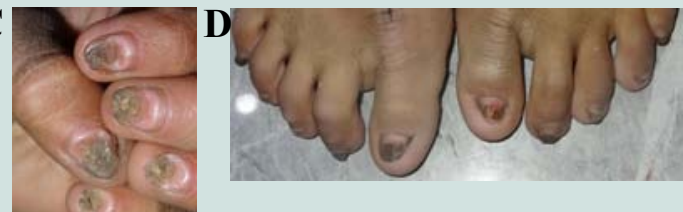

$\mathbf{E}$

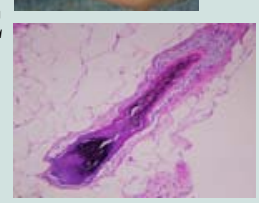

Figure 1:

(a): Case 1 with total alopecia, absence of eye lashes, and eye brows. (b): Close up view of the scalp of case 1 , small black hairs are visible within the hair follicles.

(c): Finger nails of the left hand of case 1 , all are distally dystrophic and sloping.

(d): Small, distally dystrophic and sloping toe nails of case 1.

(e): Histology of the scalp biopsy from case 1, showing a dystrophic disorganized hair follicle. (H\&E, x40). 


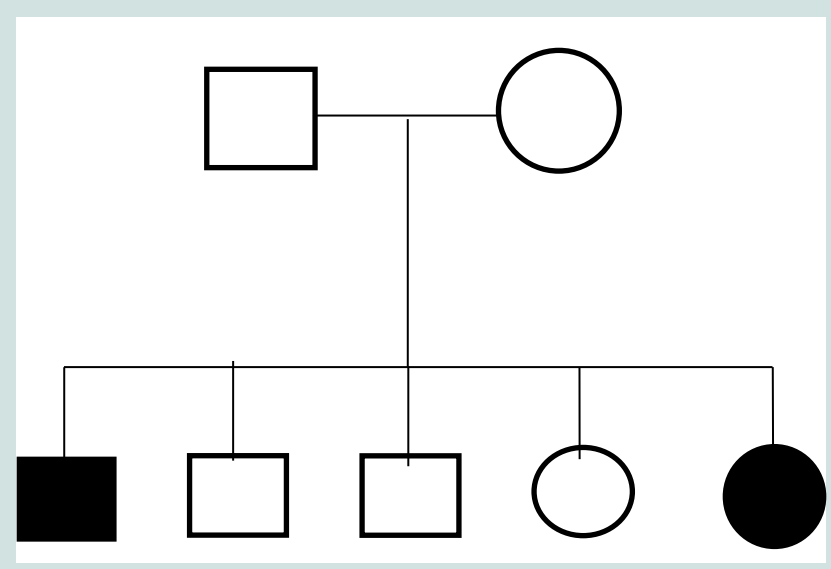

Figure 2: Pedigree of the patients.
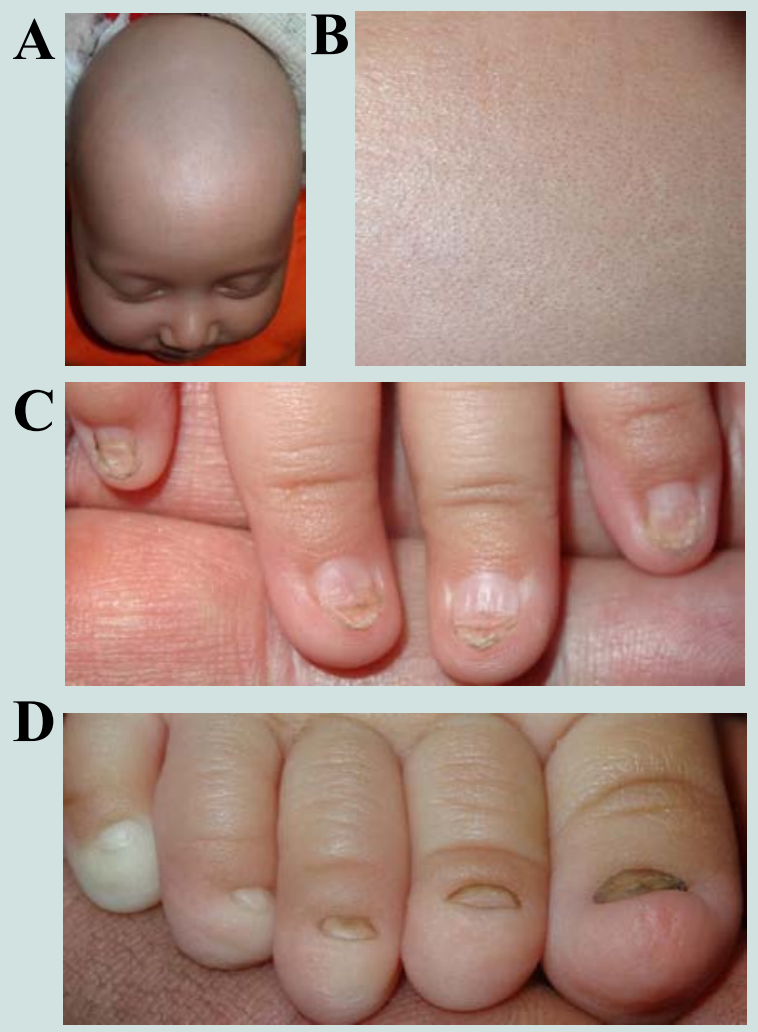

Figure 3: (a) Case 2 with total alopecia, absence of eye lashes, and eye brows.

(b) Close up view of the scalp of case 2. Tinny black hairs are visible inside the hair follicles.

(c) Distally dystrophic finger nails of right hand of case 2.

(d) Micronychia involving all toenails of right foot of case 2 .

two types: type I, having their genes on chromosome 17, and type II, having their genes on chromosome 12 .

The clinical findings in our two cases are very similar to the case described by Naeem et al. who found a mutation in the hair matrix and cuticle keratin KRTHB5 gene [10]. The histopathological findings of the scalp biopsy are similar to the case published by Lin et al. [9]. A genetic analysis would be of great help to determine the causative mutation in our case, but unfortunately this sophisticated tool is not available in most of the developing countries.

\section{Conclusion}

The two reported cases were diagnosed based on clinical findings, which were exactly the same as of the cases reported by Naeem et al. supported by the histopathological findings of the scalp biopsy, which 
Citation: Alshami MA, Mohana MJ, Alshami AM. Two Cases of Pure Hair Nail Ectodermal Dysplasia in Two Yemeni Siblings Two Cases of Pure Hair Nail Ectodermal Dysplasia in Two Yemeni Siblings. J Clin Investigat Dermatol. 2016;4(2): 3.

were similar to the case described by Lin et al. We hope that a genetic analysis could be available in near future in all developing countries, in order to confirm the underlying mutation in our two cases.

\section{References}

1. Thurnam $\mathrm{J}$ (1848) Two cases in which the skin, hair and teeth were very imperfectly developed. Med Chir Trans 31: 71-82.

2. Weech AA (1929) Hereditary ectodermal dysplasia (congenital ectodermal defect). Am J Dis Child 37: 766-790.

3. Pinheiro M, Freire-Maia N (1994) Ectodermal dysplasias: a clinical classification and a causal review. Am J Med Genet 53: 153-162.

4. Lamartine $J(2003)$ Towards a new classification of ectodermal dysplasias. Clin Exp Dermatol 28: 351-355.

5. Barbareschi M, Cambiaghi S, Crupi AC, Tadini G (1997) Family with "pure" hair-nail ectodermal dysplasia. Am J Med Genet 72: 91-93.

6. Calzavara-Pinton P, Carlino A, Benetti A, De Panfilis G (1991) Pili torti and onychodysplasia. Report of a previously undescribed hidrotic ectodermal dysplasia. Dermatologica 182: 184-187.

7. Shimomura Y, Wajid M, Petukhova L, Kurban M, Christiano AM (2010) Autosomal-dominant wooly hair resulting from disruption of keratin 74 (KRT74), a potential determinant of human hair texture. Am J Hum Genet 86: $632-638$

8. Shimomura Y, Wajid M, Kurban M, Sato N, Christiano AM (2010) Mutation in the keratin 85 (KRT85/hHb5) gene underlie pure hair and nail ectodermal dysplasia. J Invest Dermatol 130: 892-895.

9. Lin Z, Chen Q, Shi L, Lee M, Giehl KA, et al. (2012) Loss-of-function mutations in HOXC13 cause pure hair and nail ectodermal dysplasia. Am J Hum Genet 91: $906-911$

10. Naeem M, Wajid M, Lee K, Leal SM, Ahmad W (2006) A mutation in the hair matrix and cuticle keratin KRTHB5 gene causes ectodermal dysplasia of hair and nail type. J Med Genet 43: 274-279.

11. Harrison S, Sinclair R (2004) Hypotrichosis and nail dysplasia: a novel hidrotic ectodermal dysplasia. Australas J Dermatol 45: 103-105.

12. Rafiq MA, Faiyaz-UI-Haque M, Ud Din MA, Malik S, Sohail M, et al. (2005) A novel locus of ectodermal dysplasia maps to chromosome 10q24.32-q25.1. J Invest Dermatol 124: 338-342.

13. Naeem M, Jelani M, Lee K, Ali G, Chishti MS, et al. (2006) Ectodermal dysplasia of hair and nail type: mapping of a novel locus to chromosome 17p12-q21.2. Brit J Derm 155: 1184-1190.

14. Naeem M, John P, Ali G, Ahmad W (2007) Pure hair-nail ectodermal dysplasia maps to chromosome 12p11.1-q21.1 in a consanguineous Pakistani family. Clin Exp Dermatol 32: 502-505.

15. Rasool M, Nawaz S, Azhar A, Wajid M, Westermark, et al. (2010) Autosoma recessive pure hair and nail ectodermal dysplasia linked to chromosome 12p11.1-q14.3 without KRTHB5 gene mutation. Eur J Dermatol 20: 443-446.

16. Farooq M, Kurban M, Fujimoto A, Fujikawa H, Abbas O, et al. (2013) A homozygous frameshift mutation in the HOXC13 gene underlies pure hair and nail ectodermal dysplasia in a Syrian family. Hum Mutat 34: 578-581.

17. Raykova D, Klar J, Azhar A, Khan TN, Malik NA, et al. (2014) Autosomal recessive transmission of a rare KRT74 variant causes hair and nail ectodermal dysplasia: allelism with dominant woolly hair/hypotrichosis. PLoS One 9: e93607. 